\title{
LOSSY COLOR IMAGE COMPRESSION USING NON LINEARIZATION AND COLOR REDUCTION
}

\author{
Apoorva Hegde ${ }^{1}$, Sumitha Deepak ${ }^{2}$ \\ ${ }^{1}$ Assistant Professor, Department of Electronics \&Communication John Institute of Technology, Karnataka, India \\ ${ }^{2}$ Assistant Professor, Department of Electronics \& Communication John Institute of Technology, Karnataka, India
}

\begin{abstract}
Color Image compression is a technique used to reduce the bandwidth required and space needed to store the data when streaming the image across the network. In the modified version of the image compression and reconstruction we use a block optimization technique such as rank filtering to optimize the image. It is then followed by color reduction which is achieved by byte compression technique to achieve optimum compression. A desired amount of compression is achieved using the proposed compression technique. PSNR calculated is then compared with the previous technique. The simulation tool used is MATLAB to implement this algorithm.
\end{abstract}

Keywords: Image Compression, Rank Filtering, Color Reduction And Byte Compression.

\section{INTRODUCTION}

A two dimensional representationl of three dimensional object is called an image. It provides the human eyes with visual information such as color depth and contrast [1]. Digital images are image stored in the computers which are obtained by sampling and quantizing the information at regular intervals. It is basically a two dimensional array of pixels [2].

Digital images occupy alarge amount of storage space. Color images with a resolution of $1024 \times 1024$ picture elements (pixels) with 24 bits per pixel would require $3.15 \mathrm{M}$ bytes in uncompressed form. With the increasing growth in multimedia applications over a network, online data compression has become a necessity [2]. In order to increase the transmission rate the size of images need to be decreased this is can be done using image compression. Compression hardware having throughput that can match the capacity of high-speed data communication channel is the backbone of such on-line communication. This can be achieved by designing the image compression and decompression algorithms [1], [2].

Compression of images is significantly different from compressing raw binary data. In general compression programs can be used to compress images, but the result is less than optimal because images have certain statistical properties, which can be exploited by encoders specifically designed for them [2]. When it comes to images, they have finer details which are not significant and can be sacrificed to reduce the usage of bandwidth and storage space. This property is utilized in most of the compression schemes.

A great amount of redundancy is present due to lesser variation of adjacent pixels in an image. Image compression basically reduces this irrelevance and redundancy so that the image can be stored in an efficient way. Another property of high correlation between the pixels in images has been exploited for image compression. There are basically two types of redundancies defined. They are statistical and spatial redundancies [3]. Spatial redundancies deal with correlation between the neighboring pixels and spectral redundancy deals with correlation between different color planes. Thus the basic principle is to decorrelate the pixels and encode the image for transmission and storage [3].The efficiency of an image compression depends on the speed at which we can use the spatial and spectral redundancy. Images are usually represented by an 8 bit color or 24 bit color. An image basically uses either an 8 bit or 24 bit color. An 8 bit color is defined up to 256 colors forming a palette for the image, each color denoted by an 8 bit value. A 24-bit colorschemeuses 24 bits per pixel and provides a much better set of colors where each pixel is represented by three bytes corresponding to red, green and blue [4]. There are two types of compression techniques that is lossless and lossy image compression.

In lossless color image compression there is no loss of information and the exact content of the original image is preserved and after decompression the exact data can be retrieved. In lossycolor image compression a part of the original data is discarded in order to reduce file size [5]. There is degradation in quality of the image but if the process is good the loss in quality cannot be seen due to the limitation of human eyes and ears and also because of the tendency of the human senses to bridge the gaps in information [6]. This image compression algorithm basically deals with digital box filtering in section 2.1 and byte compression in section 2.2. Section 3 basically deals with performance parameters which are used to indicate the efficiency of the algorithm. The results of the experiments conducted are tabulated and given in section 4 along with conclusion given in section 5. Thus a lossy color image compression is obtained without degradation of quality. 


\section{PROPOSED ALGORITHM}

In the proposed algorithm the image is subjected to rank filtering to smoothen the image. This is applied to each of the color planes individually and then color reduction is performed by applying the byte compression technique to each pixel in an image. At the decoding end color expansion is done by adding bits to each pixel in an image to get back the original image. The image obtained after reconstruction is visually lossless without degrading the quality of the image.

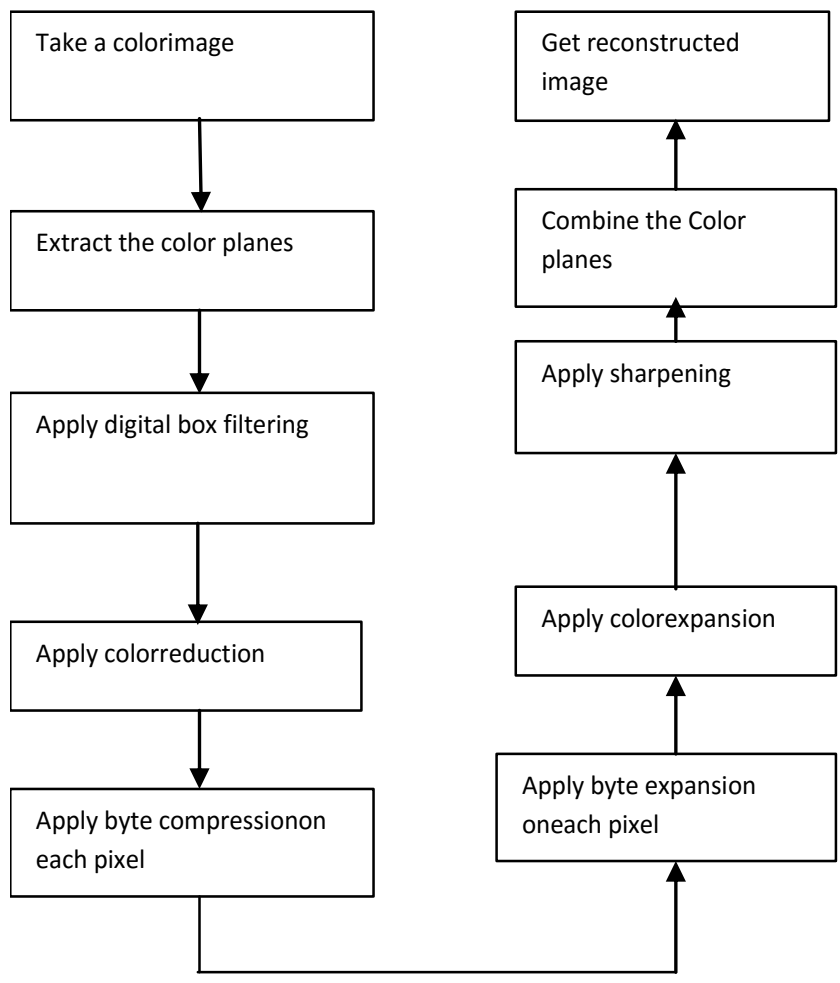

Fig 1. Proposed Algorithm

\subsection{Non Linearization}

An image has a high correlation between neighboring pixels. Rank filters are nonlinear filters and are used to compute the filtered value [7]. This is done by ranking the pixels in the increasing order of their brightness. Then the median from this sorted list is computed as the new pixel value for the processed image. As in the case of the masking operations, this is used to produce a new image and only the original pixel values are used in the ranking for the surrounding area around each of the pixel.

Median filters are used to reduce noise and smoothen an image, similar to the average filter. This technique is better than the average filter since it preserves finer details of the image [8]. The basic idea for byte compression is derived from the concept of block optimization [5]for compressing images. The important featuresof medianfilters are that it can be used to filter small, unwanted features in images. Consider a30 $\times 30$ image for which a mask of size $5 \times 5$ is applied. The filter used is a simple median filter, depending on the requirement. The procedure used is as follows.
1. Median filtering takes each pixel in an image. This is done by placing a mask which slides pixel by pixel over the entire image.

2. The median is calculated by first sorting all the values in the mask in the increasing order of their brightness.

3. The pixel then considered is replaced by the median value.

4. If the pixels considered by the mask consists of even number of pixels then the average of the two central pixel values are taken [3].

\subsection{Color Reduction And Byte Compression}

Color image consists of three planes red green and blue. When we consider 24 bit color images each pixel has 24 bits where red has eight bits, green has eight bits and blue has eight bits per pixel [3]. Hence three bytes are required to store red green and blue components.Hence color reduction is used to reduce the amount of color in an image. Byte compression is a technique used to reduces the amount of storage of RGB components from three bytes to two bytes[5].In an image most of the information is present in the least significant bit[3].Hence byte compression reduces the information in the MSB such that the visual information lost is minimal. This is a lossy compression technique.The process is as follows:

1. Each pixel in RGB color images consists of three bytes which are red, green and blue component. In order to reduce the number of bytes here each byte undergoes a division by eight resulting in a reduction of bits.

2. The same process is performed on the green component of image e that it undergoes a reduction by two bits

3. The third byte is then reduced by three bits to obtain a total reduction by 2 bytes from three.

The byte compression technique uses the shift operation for bitwise calculation. A bitwise logical right shift operation is performed where the bits shifted to the right are discarded and zeros are shifted in to the left and placed in the most significant bit.This process is suitable for unsigned numbers. In this proposed algorithm each of the components areobtained from each pixel of the color image and a shifting operation is performed on each component.

\section{PERFORMANCE PARAMETERS}

\subsection{Peak Signal to Noise Ratio}

Peak Signal to Noise ratio often termed as PSNR is defined as the ratio between maximum possible power of signals and the power of noise that affects the fidelity of its representation [3]. The range of signals is very large; hence this parameter is expressed in terms of the logarithmic decibel scale. It is most commonly used in lossy image compression to measure the quality of the reconstructed 
image. It gives an approximation of the human perception to the quality of reconstruction of the image. A higher value of PSNR indicates that the quality of the image is better.

$P S N R=20 \log \left(\frac{255}{R M S E}\right)$

Where RMSE is given as

$$
R M S E=\sqrt{ }\left(\frac{1}{M N} \times \sum_{i=1}^{M} \sum_{j=1}^{N}\left(f_{k}(i, j)-f_{k}^{\wedge}(i, j)\right)^{2}\right)
$$

Where $M$ are the number of rows and $N$ is the number columns $f_{k}(i, j)$ is the original matrix and $f_{k}^{\wedge}(i, j)$ is the output image matrix and $k$ indicates red, green and blue.

\subsection{Compression Ratio}

It indicates the amount compression that has taken place. It is defined as the ratio of the data present in the original image and the data obtained after the compression. Higher the ratio more is the degradation of images.

Compression Ratio $=\frac{\text { Uncompressed Size }}{\text { Compressed Size }}$

\section{EXPERIMENTALRESULTS, PERFORMANCE COMPARISONS}

The proposed color image compression algorithm was simulated using Matlab software version R2007b.Standard test images in ppm formats and resolution of $512 \times 512$ and $720 \times 576$ pixels downloaded from Computer vision website and USC-SIPI databaseA precision of 24 is assumed which indicates that the number of bits per pixel is 24 . In the proposed color image compression algorithm, we perform non linearization followed by color reduction using byte compression. It is compared with the existing simple basic JPEG algorithm and the existing BOBC method using linearization technique with the performance parameters such as PSNR and the compressed size as shown in Table. I. The Table. I indicate that the compressed sizes obtained using the proposed algorithm for the three images considered in the The PSNR of the images tabulated with respect to the JPEG algorithm is comparatively less due to the increase in compression because as the compression ratio increases the value of PSNR decreases. But the quality of the image is good, even though the amount of compression achieved is not as good as JPEG .Hence we can say that it is visually lossless [7].

Consider an image of mandrill of size $512 \times 512$ shown in Fig.2given as input to the proposed algorithm .The reconstructed image obtained is of considerable good quality and is visually lossless as shown in Fig.3

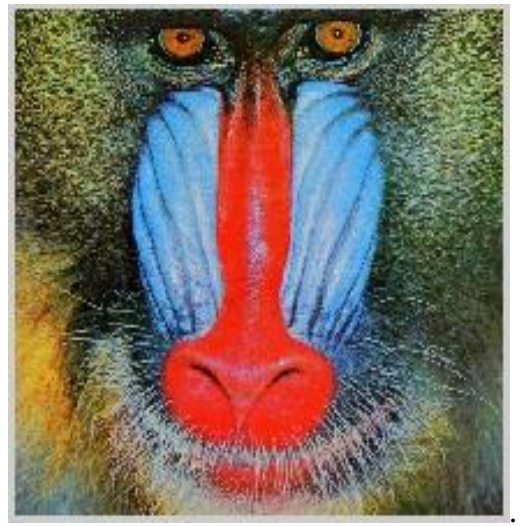

Fig-2: Original Image

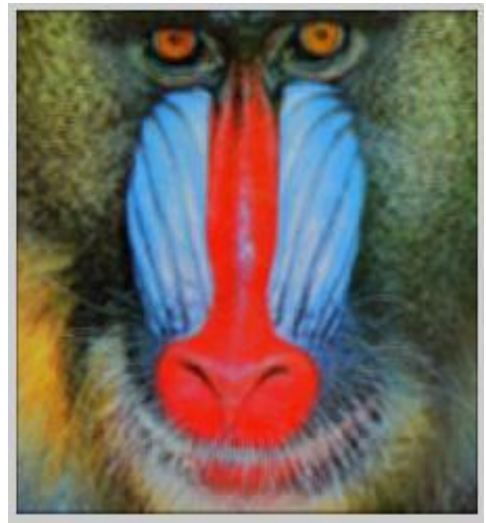

Fig-3: Reconstructed Image

\begin{tabular}{|l|l|l|l|l|l|l|}
\hline \multicolumn{6}{|c|}{ TABLE 1: Compressed Sizes \&PSNR of Images } \\
\hline $\begin{array}{l}\text { Sl. } \\
\text { no }\end{array}$ & $\begin{array}{l}\text { Ima } \\
\text { ges }\end{array}$ & $\begin{array}{l}\text { Ori } \\
\text { gina } \\
\text { I } \\
\text { Ima } \\
\text { ge } \\
\text { Size } \\
\text { (KB }\end{array}$ & $\begin{array}{l}\text { Compres } \\
\text { for } \\
\text { BOBC } \\
\text { using } \\
\text { linearizat } \\
\text { ion(KB) }\end{array}$ & $\begin{array}{l}\text { Compres } \\
\text { sed Size } \\
\text { for } \\
\text { BOBC } \\
\text { using } \\
\text { non } \\
\text { linearizat } \\
\text { ion(KB) }\end{array}$ & $\begin{array}{l}\text { PS } \\
\text { NR } \\
\text { in } \\
\text { dB }\end{array}$ & $\begin{array}{l}\text { PS } \\
\text { NR } \\
\text { in } \\
\text { dB }\end{array}$ \\
\hline 1 & $\begin{array}{l}\text { Len } \\
\text { a }\end{array}$ & 631 & 298 & 268 & 62.4 & 64.4 \\
\hline 2 & $\begin{array}{l}\text { Boa } \\
\text { ts }\end{array}$ & 106 & 567 & 511 & 62.0 & 62.3 \\
\hline 3 & $\begin{array}{l}\text { Bar } \\
\text { bara }\end{array}$ & 107 & 558 & 519 & $\begin{array}{l}54.1 \\
218\end{array}$ & $\begin{array}{l}64.0 \\
56\end{array}$ \\
\hline 4 & $\begin{array}{l}\text { Pep } \\
\text { per }\end{array}$ & 658 & 319 & 291 & 59.8 & 63.4 \\
& & & 40 & 87 \\
\hline
\end{tabular}

\section{CONCLUSION}

This modified proposed algorithm is suitable for most still photo images. It provides considerable amount of compression without degrading the quality of the images. The main advantage of this compression scheme is that it provides a considerable amount of compression and is used for a wide range of images. This compression scheme can be compared with other compression techniques in terms of compression ratio and corresponding quality [5]. 


\section{FUTURE SCOPE}

Image Compression technique will be useful for a variety of applications were still images are required. It can be used to compress to high quality images which require a large amount of free space[9]. It can be modified to be used for compressing medical images using modern technologies. It can help to provide a mapping from three dimensional spaces to two dimensional spaces thus increasing its value effectively [9].

\section{REFERENCES}

[1]. S. Bhattacharjee, S. Das, D. Roy Choudhury and P. Pal Chouduri, "A Pipelined Architecture Algorithm for Image Compression",Proceedings Data Compression Conference, Salt lake City, USA, March 1997.

[2]. Rafael C. Gonzalez, Richard E. Woods, "Digital ImageProcessing", Pearson Education, 2nd Edition, 2002.

[3]. Alasdair McAndrew "An Introduction to DigitalImageProcessing with MATLAB"School of Computer Science andMathematics Victoria University of Technology.

[4]. Jorg Ritter and Paul Molitor "A pipelined architecture for partitioned DWT based lossy image compression using FPGA's", International Symposium on FPGA, pages 201-206, 2001.

[5]. [5].AmiyaHalder, SouravDey, Soumyodeep Mukherjee and Ayan Banerjee, "An Efficient Image Compression Algorithm Based on Block Optimization and Byte Compression", ICISA-2010, Chennai, Tamilnadu, India, pp.14-18, Feb 6, 2010.

[6]. AmiyaHalder, Dipak Kumar Kole and SubarnaBhattacharjee, "Online ColourImage Compression based on PipelinedArchitecture" ICCEE2009, Dubai, UAE.

[7]. Jose Oliver, Otoniel Lopez, M. Martínez-Rach, Pablo Piñol,Carlos.T.Calafate1, Manuel P Malumbres, "Overview on Image Compression", Technical University of Valencia, Spain Miguel Hernandez University, Spain.

[8]. AmiyaHalder, SouravDey, Soumyodeep Mukherjee and Ayan Banerjee, "An Efficient Image Compression Algorithm Based on Block Optimization and Byte Compression", ICISA-2010, Chennai, Tamilnadu, India, pp.14-18, Feb 6, 2010.

[9]. S Jayaraman,SEsakkirajan,TVeerakumar, "Digital Image Processing", Tata McGraw Hill Education Pvt Ltd, Seventh Edition, 2007.

\section{BIOGRAPHIES}

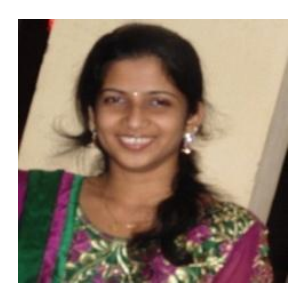

ApoorvaHegde: received her B.E degree from P.A College of Engineering and her MTECH degree from NMAMIT NitteKarkala .She has worked as an Assistant Professor in Canara Engineering College, Mangalore. She is now currently working as an Assistant Professor in the Electronics and communication department at TJohn Institute of technology Bangalore. Her areas of research interests include Medical image processing, digital communication and antenna design.

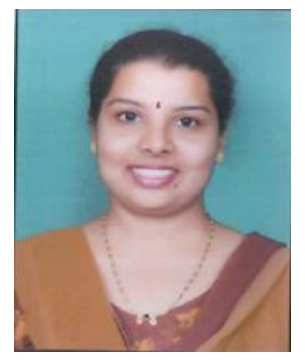

Sumitha Deepak, post graduated from RV College of Engineering, Bangalore, has 8 years of teaching experience in NITK, Surathkal, Karnataka, DayanandSagar College of Engineering, Bangalore and PESIT, Bangalore. She is currently serving as an Asst. Professor at T.John Institute of Technology, Bangalore. Her areas of research interests include Digital Communication and Antenna Design. 